\title{
Early Blindness Shapes Cortical Representations of Auditory Frequency within Auditory Cortex
}

\author{
Elizabeth Huber, ${ }^{1}$ Kelly Chang, ${ }^{1}$ IVan Alvarez, ${ }^{2}$ Aaron Hundle, ${ }^{2}{ }^{-}$Holly Bridge, ${ }^{2}$ and $\oplus^{-I o n e ~ F i n e ~}{ }^{1}$ \\ ${ }^{1}$ University of Washington, Seattle, Washington 98195, and ${ }^{2}$ University of Oxford, Oxford OX1 2JD, United Kingdom
}

Early loss of vision is classically linked to large-scale cross-modal plasticity within occipital cortex. Much less is known about the effects of early blindness on auditory cortex. Here, we examine the effects of early blindness on the cortical representation of auditory frequency within human primary and secondary auditory areas using fMRI. We observe that 4 individuals with early blindness ( 2 females), and a group of 5 individuals with anophthalmia ( 1 female), a condition in which both eyes fail to develop, have lower response amplitudes and narrower voxelwise tuning bandwidths compared with a group of typically sighted individuals. These results provide some of the first evidence in human participants for compensatory plasticity within nondeprived sensory areas as a result of sensory loss.

Key words: auditory; blindness; neural plasticity; population receptive field; visual deprivation

\section{Significance Statement}

Early blindness has been linked to enhanced perception of the auditory world, including auditory localization and pitch perception. Here we used fMRI to compare neural responses with auditory stimuli within auditory cortex across sighted, early blind, and anophthalmic individuals, in whom both eyes fail to develop. We find more refined frequency tuning in blind subjects, providing some of the first evidence in human subjects for compensation within nondeprived primary sensory areas as a result of blindness early in life.

\section{Introduction}

It has long been held that early blindness leads to enhanced perception of auditory stimuli, and a number of behavioral studies have linked early-onset blindness to superior pitch perception (Gougoux et al., 2004; Wan et al., 2010; Voss and Zatorre, 2012). However, the neural basis of any such enhancements is not known. Heightened frequency discrimination as a result of blindness early in life could potentially be mediated by compensatory plasticity within deprived occipital cortex. Indeed, Kujala et al. (1992) observed that neural responses to pure tones, measured using EEG, were shifted posteriorly in a group of blind subjects, and subsequent MEG measurements further suggested these responses might be localized to occipital cortex (Kujala et al., 1995). Using fMRI, Watkins et al. (2013) observed responses to pure tones within area $\mathrm{hMT}^{+}$, an area typically associated with visual motion perception, in a subset of individuals with anophthalmia.

\footnotetext{
Received Nov. 11, 2018; revised April 3, 2019; accepted April 4, 2019.

Author contributions: E.H., I.A., A.H., and H.B. performed research; E.H., K.C., A.H., and I.F. analyzed data; E.H. wrote the first draft of the paper; E.H., K.C., I.A., H.B., and I.F. edited the paper; H.B. and I.F. designed research.

We thank Geoffrey M. Boynton for useful comments and suggestions.

The authors declare no competing financial interests.

Correspondence should be addressed to Kelly Chang at kchang4@uw.edu.

Extended Data is available at https://www.jneurosci.org/content/39/26/5143/tab-figures-data\#figdatasupplementary-materials.

https://doi.org/10.1523/JNEUROSCI.2896-18.2019

Copyright $\odot 2019$ the authors
}

More recently, Huber et al. (2019) demonstrated that responses with $\mathrm{hMT}^{+}$are selective for both auditory frequency and motion as a result of early blindness.

Enhanced auditory performance as a result of blindness might also be mediated by plasticity within auditory areas. Previous MEG results in congenitally blind individuals suggested an expanded tonotopic representation within a region identified as primary auditory cortex (Elbert et al., 2002), with responses to 500 and $4000 \mathrm{~Hz}$ tones being separated more widely on the cortical surface in blind relative to sighted individuals. An expanded tonotopic map might permit a finer frequency resolution. Indeed, expansion in the representation of specific auditory frequencies has been linked to superior discrimination performance after extensive training (Recanzone et al., 1993). However, a few studies have reported an attenuated response to pure tone auditory stimuli in the temporal lobe of early blind individuals (Gougoux et al., 2009; Stevens and Weaver, 2009; Watkins et al., 2013). This has been interpreted as reflecting either increased "efficiency" of processing within the intact modality (Gougoux et al., 2009; Stevens and Weaver, 2009) or reduced participation in auditory processing, perhaps due to function being "usurped" by reorganized occipital cortex, as may occur for auditory motion processing (Jiang et al., 2014; Dormal et al., 2015).

Here, we used fMRI and population receptive field (pRF) modeling (Dumoulin and Wandell, 2008) in acoustic frequency 
Table 1. Subject demographics and clinical descriptions

\begin{tabular}{|c|c|c|c|c|c|}
\hline Subject no. & Sex & Location & Stimuli & Age (yr) & Clinical description \\
\hline EB01 & Male & University of Washington & Stationary/moving & 32 & Leber's congenital amaurosis, low light perception \\
\hline EB02 & Female & University of Washington & Stationary/moving & 38 & $\begin{array}{l}\text { Retinopathy of prematurity, no light perception in right eye, minimal peripheral light } \\
\text { perception in left eye, } 3 \text { months premature }\end{array}$ \\
\hline EB03 & Female & University of Washington & Stationary/moving & 55 & $\begin{array}{l}\text { Retinopathy of prematurity, low light perception until retina detached at age } 25 \text {, } \\
2 \text { months premature }\end{array}$ \\
\hline EB04 & Male & University of Washington & Stationary/moving & 52 & Congenital glaucoma, no light perception \\
\hline AN01 & Male & Oxford & Moving & 36 & Isolated bilateral anophthalmia \\
\hline AN02 & Female & 0xford & Moving & 39 & Isolated bilateral anophthalmia \\
\hline AN03 & Male & 0xford & Moving & 26 & Isolated bilateral anophthalmia \\
\hline AN05 & Male & Oxford & Moving & 30 & Isolated bilateral anophthalmia \\
\hline AN06 & Male & 0xford & Moving & 31 & Isolated bilateral anophthalmia \\
\hline
\end{tabular}

space (Thomas et al., 2015) to characterize the effects of blindness on primary auditory cortex within a group of individuals with early blindness, including a subset of individuals with anophthalmia, in whom the eyes fail to develop. In blind individuals, we see evidence for a more refined representation of auditory frequency within auditory cortex.

\section{Materials and Methods}

\section{Participants}

Table 1 provides subject details. Data were collected at two research sites: the University of Washington (UW; Seattle) and Oxford University (Oxford, UK). At the UW, data were collected using two protocols (static and motion, described below). Four early blind individuals ( 2 females) and four age-matched controls (static: 2 females; motion: 3 females) completed both the static and motion protocols. Two control subjects were repeated across protocols, whereas the remaining control subjects completed one protocol or the other, due to scheduling constraints.

At the University of Oxford, data were collected using the motion paradigm with participants with anophthalmia $(N=5,1$ female $)$ and an age-matched control group ( $N=9,3$ females). All subjects reported normal hearing, and no history of neurological or psychiatric illness.

At each site, the study was approved by the Institutional Review Board, and all subjects provided written informed consent.

\section{$M R I$}

Overhead lighting was dimmed during MRI acquisition, and subjects were instructed to keep their eyes closed throughout testing. Foam padding was used to minimize head motion. A closed-circuit camera system was used to monitor compliance, and all subjects successfully followed instructions and kept eyes closed during all functional scans.

\section{Acquisition protocol}

UW data were collected using a 3T Achieva scanner (Philips) at the UW Diagnostic Imaging Sciences Center using a 32-channel head coil. In the static session, four functional scans were acquired using a standard EPI sequence ( 36 slices, TR/TE $=2000 / 25 \mathrm{~ms}$, flip angle $=$ $80^{\circ}$, no slice gap). After discarding the first 5 timeframes, each scan consisted of 255 volumes at an effective voxel size of $3.00 \mathrm{~mm}^{3}$ isotropic $\left(\mathrm{FOV}=240 \times 240 \times 108 \mathrm{~mm}^{3}\right.$, matrix size $\left.=80 \times 80 \times 36\right)$. In the motion session, 4 functional scans were acquired using a standard EPI sequence (30 slices, TR/TE $=2000 / 25 \mathrm{~ms}$, flip angle $=80^{\circ}$, no slice gap). After discarding the first 5 timeframes, each scan consisted of 144 volumes at an effective voxel size of $2.75 \times 2.75 \times 3.00$ $\mathrm{mm}^{3}$ ( $3 \mathrm{~mm}$ in-plane; FOV $=220 \times 220 \times 90 \mathrm{~mm}^{3}$, matrix size $=$ $80 \times 80 \times 30)$.

Oxford data were acquired with a $7 \mathrm{~T}$ scanner (Siemens) at the Oxford Centre for Functional MRI of the Brain using a 32-channel head coil. In each session, six functional scans were acquired using a multishot EPI sequence $(64$ slices, $\mathrm{TR} / \mathrm{TE}=2488 / 27.8$, flip angle $=$ $85^{\circ}$, no slice gap) with in-plane acceleration using parallel imaging $($ GRAPPA factor $=2)$ (Griswold et al., 2002) and through-slice accel- eration using multiband imaging $(\mathrm{MB}$ factor $=2)$ (Moeller et al., 2010). Each scan consisted of 116 volumes at an effective voxel size of $1.2 \mathrm{~mm}^{3}$ isotropic $\left(\mathrm{FOV}=192 \times 192 \times 76.8 \mathrm{~mm}^{3}\right.$, matrix size $=$ $160 \times 160 \times 64)$.

\section{Auditory stimulus creation}

Auditory stimuli were generated in MATLAB (The MathWorks) using the Psychophysics Toolbox (www.psychtoolbox.org) and custom MATLAB software, and were delivered via insert earphones. Auditory stimuli were delivered using MR-compatible Sensimetrics S14 earphones (Sensimetrics) at both research sites.

All stimuli were presented at a sampling rate of $44.1 \mathrm{kHz}$. Auditory calibration was performed using a standard, two-step procedure. We began by compensating for frequency-specific distortions in the earphone output, adjusting stimulus intensities to ensure flat frequency transmission from $100 \mathrm{~Hz}$ to $8 \mathrm{kHz}$. Next, stimulus sound intensities were adjusted according to a standard equal-loudness curve created for insert earphones (ISO 226) to approximate equal perceived loudness across frequency. Actual sound intensities (65-83 dB SPL) were matched to the perceived loudness of a $1 \mathrm{kHz}$ tone (reference frequency) at $70 \mathrm{~dB}$ SPL. Acoustic noise from the scanner was attenuated by expanding-foam ear-tips, as well as protective earmuffs placed over the ear following earphone insertion. Subjects confirmed that all tones were clearly audible, and of approximately equal loudness across the frequency range.

\section{Auditory stimulus and task}

As described in Table 1, all 4 early blind UW subjects participated in both the static and the motion protocols. All 5 Oxford anophthalmic subjects only participated in the motion protocol.

Motion stimuli. Stimuli consisted of bandpass noise burst pairs centered at each of 7 frequencies $(100-3162 \mathrm{~Hz}$, stimulus bandwidth of $\sim 0.8$ octaves), presented in pseudo-randomized blocks that lasted $2 \mathrm{~s}$. The auditory motion within each block was simulated using interaural time difference and consisted of a pair of identical $1 \mathrm{~s}$ bursts (treated as a single event) traveling at $30 \mathrm{~m} / \mathrm{s}$ from left to right, or vice versa, along a fronto-parallel plane $10 \mathrm{~m}$ in front of the listener. Subjects were asked to monitor the stimulus frequency and report via button press each time the same exact frequency-band noise burst pair was repeated ( 1 - back, $10 \%$ of trials). The task ensured that the subjects maintained attention for the duration of the scan. Catch trials were included in all analyses.

Static stimuli. Stimuli were blocks of pure tone bursts, which varied in auditory frequency $(88-8000 \mathrm{~Hz}$, sampled in half-octave steps). Each $2 \mathrm{~s}$ stimulus block contained eight pure tone bursts of the same frequency. Tone durations were alternated in pseudorandomized order, switching durations at least 4 times during each $2 \mathrm{~s}$ block, resulting in a "Morse code"-like pattern of long and short tones, which served to increase the perceptual salience of the stimuli over the regular pattern of background scanner noise (Da Costa et al., 2011; Thomas et al., 2015).

Each run consisted of a series of sequences of 3-4 ascending or descending half-octave steps, with the starting frequency and sequence (ascending vs descending) selected pseudo randomly at the beginning 
of each sequence. The pseudo randomization ensured that the distribution of presented frequencies between 88 and $8000 \mathrm{~Hz}$ was uniform, and that there were equal numbers of ascending and descending sequences. Subjects were asked to monitor the stimulus frequency and report via button press each time the same exact frequency was repeated ( 1 - back, $10 \%$ of trials). The task ensured that the subjects maintained attention for the duration of the scan. Catch trials were included in all analyses.

\section{MR data preprocessing}

Standard preprocessing of $\mathrm{fMRI}$ data was performed using BrainVoyager QX software (version 2.3.1, Brain Innovation), including slice scan time correction, 3D motion correction, and temporal high-pass filtering (cutoff: 2 cycles). Parameters generated during motion correction were recorded to ensure that subject motion within a scan did not account for pRF tuning parameters, as described below.

Functional data were aligned to the T1-weighted anatomical image acquired in the same session (MPRAGE, $1 \mathrm{~mm}^{3}$ ) and resampled to 3 $\mathrm{mm}^{3}$ resolution. The BrainVoyager QX automatic segmentation routine was used to reconstruct the cortical surface at the white-gray matter border (with hand-editing to minimize segmentation errors) and the resulting smooth 3D surface was partially inflated.

\section{ROI selection}

Auditory cortex borders were drawn generously based on purely anatomical criteria to include all voxels within a contiguous region of auditory cortex between the lateral border on the crown of the superior temporal gyrus, the medial border within the fundus of the lateral sulcus, the posterior border of the supramarginal gyrus, and the anterior border of the most anterior portion of the temporal lobe.

The primary auditory cortex (PAC) ROI was defined using a combination of anatomical and functional criteria (Thomas et al., 2015). For simplicity, we refer here to the pair of tonotopic gradients comprising $\mathrm{hAl}$ and $\mathrm{hR}$ as PAC. In primates, the auditory core area contains up to three tonotopic subdivisions, A1, R, and RT (Hackett et al., 1998; Hackett, 2008), which have been localized to the medial two-thirds of Heshl's gyrus (Rademacher et al., 2001; Dick et al., 2012). Human neuroimaging studies have identified at least two tonotopic gradients, presumed to be the human homologs of areas A1 and R (Formisano et al., 2003; Humphries et al., 2010; Da Costa et al., 2011, 2015; Striem-Amit et al., 2011; Langers and van Dijk, 2012; Moerel et al., 2012; Thomas et al., 2015). We identified PAC as consisting of two mirror-symmetric tonotopic gradients, meeting at a low-frequency reversal on the crown of Heschl's gyrus. Based on this tonotopic map and the underlying anatomy, for each subject, a PAC ROI was defined as a contiguous patch of cortical surface spanning Heschl's gyrus and containing both tonotopic gradients. Anterior and posterior borders were drawn along the outer high-frequency representations of the tonotopic maps, and lateral and medial borders were conservatively drawn to include only the medial two-thirds of Heschl's gyrus. Although this ROI selection method cannot isolate auditory core from auditory belts areas that share the same tonotopic gradients, it does provide a consistent PAC definition for individual subjects across distinct acquisition and stimulus types (Da Costa et al., 2015; Thomas et al., 2015).

Large regions outside PAC also show evidence of frequency tuning. However, because there is still uncertainty about how tuning profiles relate to subdivisions of secondary auditory areas (Schönwiesner and Zatorre, 2009; Barton et al., 2012; Moerel et al., 2014; Santoro et al., 2017), we treat regions outside PAC as a single ROI. Our secondary auditory areas ROI was simply defined as voxels within the auditory cortex ROI that were not within PAC.

\section{Population receptive field analysis}

Preprocessed fMRI data were analyzed using methods described in detail in Thomas et al. (2015). In brief, we assumed a Gaussian sensitivity profile of unit amplitude on a log auditory frequency axis for each voxel. Using custom software written in MATLAB, we found, for each voxel, the center $\left(f_{0}\right)$ and SD $(\sigma)$ of the Gaussian that when multiplied by the stimulus over time and convolved with an HRF (see below) produced a predicted time course that best correlated with the voxel's measured time course. For each voxel, the best-fitting value of $f_{0}$ is interpreted as that voxel's preferred frequency, while the best-fitting value of $\sigma$ is interpreted as the tuning bandwidth.

Based on Boynton et al. (1996), we modeled the auditory HRF as a gamma function, with initial parameters $n=3$, tau $(\tau)=1.5$, and delay $(\delta)=1.8$. We then used an iterative procedure to obtain individual HRF estimates. For each subject, we iteratively looped three times between two steps: (1) optimizing $f_{0}$ and $\sigma$ while holding HRF parameters fixed, (2) optimizing $\tau$ and $\delta$ while holding $f_{0}$ and $\sigma$ fixed. This HRF optimization was only performed on a subset of the data, using voxels with an initial fitted correlation value $>0.25$. Median $\tau$ and $\delta$ parameters were used to approximate each individual's HRF across the entire auditory cortex ROI. We then refit the pRF parameters $\left(f_{0}\right.$ and $\sigma$ ) for all voxels within the auditory cortex ROI, using these individually fitted HRF parameters. After finding the best fitting values of $f_{0}$ and $\sigma$, we used linear regression to find the amplitude value, $a$, that minimized the root mean square error between predicted and obtained time courses.

After fitting, only voxels that met the following criteria were retained for further analysis: (1) the correlation between the observed fMRI time course and the time course predicted by the best-fitting pRF (our goodness-of-fit index) was $>0.20$; $(2)$ the center $\left(f_{0}\right)$ of the best fitting pRF fell within the range of tested values $(44-16,000 \mathrm{~Hz}$ for the static stimulus; and $50-6324 \mathrm{~Hz}$ for the motion stimulus); and (3) the SD $(\sigma)$ of the best fitting pRF was $>0.1 \log$ (base 10) units and $<2 \log$ units. For comparison, the presented frequencies in the static condition were separated by 0.15 log units, and each motion band center was separated by $0.25 \log$ units. (4) Because amplitude values were positively skewed, we identified pRF fits with outlying amplitude values nonparametrically. We excluded voxels where the amplitude of the best fitting pRF did not fall between $(\mathrm{Q} 1-3 \times \mathrm{IQR})$ and $(\mathrm{Q} 3+$ $3 \times \mathrm{IQR}$ ), where $\mathrm{Q} 1$, and $\mathrm{Q} 3$ refer to the first and third quartiles, and IQR is the interquartile range. Within PAC, acceptable amplitudes fell between 0 and 6.1414 (6.8945\% of voxels excluded). Within secondary cortex, acceptable amplitudes fell between 0 and 5.4163 (3.2319\% voxels excluded).

\section{Statistical analyses}

Estimating $p R F$ fit significance. $\mathrm{pRF}$ model fits were compared against a null model created using a randomized stimulus representation. In the case of the static stimulus, to preserve the temporal structure within individual stimulus blocks, we randomized the stimulus block order while retaining the ascending or descending series structure within each block. For each permutation, we calculated the false discovery rate based on $R>0.2, \mu, \sigma$, and $\alpha$ within acceptable ranges, as described in Materials and Methods. False discovery rates were calculated based on 100 permutations. Within auditory cortex, collapsed across all subjects and datasets; this stimulus-label permutation generated a mean false discovery rate of $11.2617 \%$ for a correlation threshold of $r>0.2$. This threshold excluded voxel responses that were clearly noise.

Group differences. Group differences were assessed using multifactor ANOVA using MATLAB and the Statistics Toolbox (Release 2016a). Datasets (UW static, UW motion, Oxford motion), group (blind vs sighted), and hemisphere (when included) were treated as independent variables. In all cases, we tested for the normality of the residuals with visual inspection and the Shapiro-Wilk test for normality.

For ANOVAs containing all three datasets, we first performed exploratory analyses using the 4 early blind and 2 sighted subjects who participated in both the static and the motion conditions as separate subjects. We then confirmed that the presence of these repeated subjects was not statistically critical by performing separate ANOVAs on the motion datasets only (which contained no repeated subjects). The results for the motion dataset only analyses are reported whenever they differed from those obtained using all three datasets.

Comparisons of pRF frequency distributions of across groups were performed using a bootstrapping procedure, based on the $\chi^{2}$ test of independence. This test compares the obtained frequency distributions for each group with those that would be obtained if the frequency distribution were independent of group assignment. Significance values were obtained by comparing the real $\chi^{2}$ values with those obtained by boot- 
strapping simulated distributions, created using random assignment of subjects across the two groups (1000 simulations).

Comparison across datasets. To minimize site-based differences, an individualized HRF was estimated for each subject before pRF fitting, as described above (Thomas et al., 2015). pRF model estimates of tuning parameters $f_{0}$ and $\sigma$ are expected to be reasonably robust to differences in SNR associated with collecting data at both Oxford and UW. Amplitude values, $a$ would be expected to differ significantly across different protocols (motion vs static), MR imaging protocols or site.

Differences between motion and static stimuli could also potentially influence estimates of $f_{0}$ and $\sigma$. For the motion stimulus, we used a more restricted stimulus set that spanned seven frequency bands, within a range of frequencies for which interaural time difference cues are equally strongly effective, so pRF estimates of $f_{0}$ could only reliably be estimated within a smaller frequency range. The reduced frequency range of the motion stimulus would also be expected to increase uncertainty in the estimate of $\sigma$, especially for smaller values. Finally, it is possible that interactions between motion sensitivity and frequency tuning could alter pRF estimates across the two stimuli due to recruitment of different neuronal populations.

\section{Results}

Figure 1 shows tonotopic maps in auditory cortex for 4 example subjects. Tonotopic organization did not differ significantly across datasets: for subjects who participated in both UW motion and UW static scans the mean voxelwise cross-correlation (Pearson's $r$ ) between the two datasets was 0.7457 in PAC and 0.4874 in secondary AC, values similar to previous studies examining replicability across different stimulus and acquisition protocols on the same UW scanner (Thomas et al., 2015). Tonotopic maps for all subjects and datasets are included in Fig. 1-1 to Fig. 1-29.

\section{Auditory cortex size}

As described in the Introduction, previous MEG results have suggested that early blindness may result in a 1.8-fold expansion of early auditory areas (Elbert et al., 2002), although a reduction in the number of frequency selective voxels in auditory cortex has also been reported (Stevens and Weaver, 2009).

We began by examining PAC size by using all the voxels within the hand-drawn PAC ROI as our dependent measure. Group differences were assessed using an ANOVA with dataset, hemisphere, and blindness as fixed effects and the number of voxels within PAC as the dependent measure. This revealed an effect of dataset $\left(F_{(2,48)}=17.45, p<0.0001\right)$, but no effect of blindness $\left(F_{(1,48)}=0.86, p=0.3579\right)$, or hemisphere $\left(F_{(1,48)}=0.02\right.$, $p=0.878)$. There was a significant interaction between blindness and dataset $\left(F_{(2,48)}=3.71, p=0.0319\right)$. A post hoc Tukey-Kramer test showed that the UW static dataset resulted in a significantly larger definition of PAC than both the UW motion and Oxford motion datasets. This might be due to a difference in scanner quality, voxel acquisition size, and/or stimuli (e.g., the wider frequency range). No other interactions were significant.

We also assessed group differences in the number of voxels which were successfully fit in PAC and secondary auditory areas $(R>0.2, \mu, \sigma$, and $\alpha$ within acceptable ranges, as described in Materials and Methods). In the early blind/anophthalmic group, the mean number of successfully fit voxels in the UW Static, UW Motion, and Oxford motion conditions were 191, 208.25, and 52.8, respectively. In the control group, the mean number of voxels were $147.75,167.5$, and 113.33, respectively. We found no evidence for an effect of blindness on the number of frequency-tuned voxels within either PAC or secondary auditory areas.

Within PAC, we once again found an effect of dataset $\left(F_{(2,48)}=9.97\right.$, $p=0.0002)$, but no effect of blindness $\left(F_{(1,48)}=0.13, p=0.7241\right)$ or hemisphere $\left(F_{(1,48)}=0.11, p=0.7461\right)$. No other interactions were significant. Within secondary auditory areas, we found an effect of dataset $\left(F_{(2,48)}=7.06, p=0.002\right)$, but no effect of blindness $\left(F_{(1,48)}=0.46 p=0.5021\right)$ or hemisphere $\left(F_{(1,48)}=\right.$ $0.19, p=0.6681)$. No interactions were significant. For both PAC and secondary auditory areas, post hoc Tukey-Kramer tests suggested that the effect of dataset was driven by a smaller number of voxels within PAC passing threshold for Oxford anophthalmic individuals. This was likely due to an interaction between reduced signal-to-noise in the Oxford dataset (due to the smaller acquisition voxel size) and lower pRF amplitudes in blind individuals, see below.

\section{HRFs}

A wide variety of studies have found metabolic differences in occipital cortex between early blind and sighted individuals (WanetDefalque et al., 1988; Veraart et al., 1990; De Volder et al., 1997; Weaver et al., 2013; Coullon et al., 2015). To examine potential differences in auditory cortex hemodynamics across blind and sighted subjects, we performed a mixed-design ANOVA with dataset and blindness as fixed effects and the time-to-peak of the estimated HRF as the dependent measure. We found no main effect of dataset $\left(F_{(2,24)}=0.08, p=0.9197\right)$, no effect of blindness $\left(F_{(1,24)}=0.21, p=\right.$ $0.6479)$, and no significant interactions on the time-to-peak of the hemodynamic function within the auditory cortex ROI.

\section{Response amplitudes}

As described in the Introduction, a number of studies report an attenuated response to pure tone stimuli versus silence in the temporal lobe of blind individuals (Gougoux et al., 2009; Stevens and Weaver, 2009; Watkins et al., 2013) when comparing responses with pure tones versus silence (GLM: sound versus silence).

As shown in Figure $2 A, C$, we find that early blind and anophthalmic participants have significantly smaller $\beta$ weights than sighted subjects, within both PAC and secondary auditory areas. For individual subjects, see Fig. 2-1 and Fig. 2-2. Within PAC using a mixed-design ANOVA with dataset, hemisphere, and blindness as fixed effects and the GLM response to sound versus silence as the dependent measure, we found no main effect of dataset $\left(F_{(2,48)}=\right.$ $1.56, p=0.2197)$, an effect of blindness $\left(F_{(1,48)}=5.63, p=0.0218\right)$, no effect of hemisphere $\left(F_{(1,48)}=0.01, p=0.9366\right)$, and no significant interactions. Within secondary auditory cortical areas, we found no main effect of dataset $\left(F_{(2,48)}=0.92, p=0.4039\right)$, a marginally significant effect of blindness $\left(F_{(1,48)}=3.66, p=0.0618\right)$, no effect of hemisphere $\left(F_{(1,48)}=0.05, p=0.824\right)$, and no significant interactions.

\section{pRF model response amplitudes}

One concern is that $\beta$ weights for pure tones versus silence might potentially reflect narrower tuning (as found for our blind individuals, see below) rather than reduced responsiveness; narrower tuning would be expected to result in a smaller region of cortex responding to any given narrowband stimulus, thereby reducing measured activation in a GLM model. An advantage of our pRF approach is that it separately represents tuning width and response amplitude. As shown in Figure $2 B, D$, pRF response amplitudes are smaller in blind versus sighted subjects within both PAC and secondary auditory cortex. For individual subjects, see Fig. 2-3 and Fig. 2-4, consistent with the GLM analysis of sound versus silence. Group differences in pRF amplitudes were assessed using an ANOVA with dataset, hemisphere, and blindness as fixed effects and pRF amplitude as the dependent measure. Within PAC, this 
A

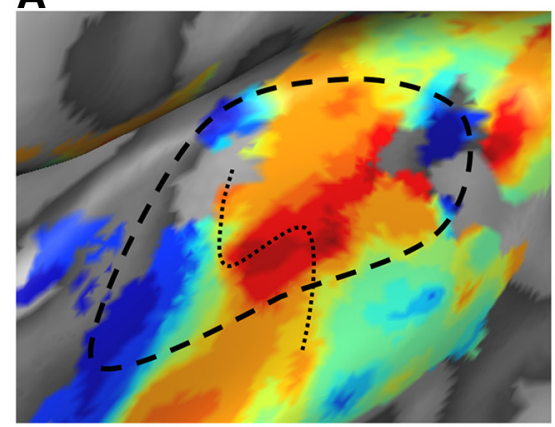

C
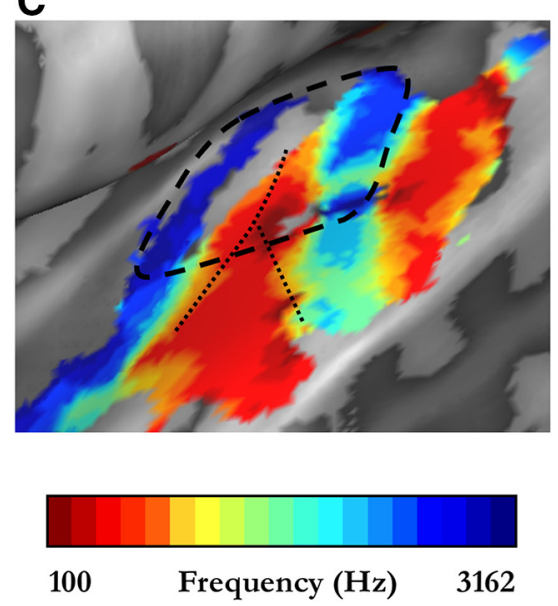

B

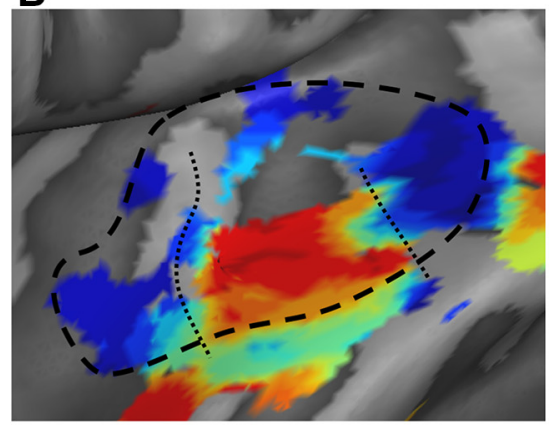

D

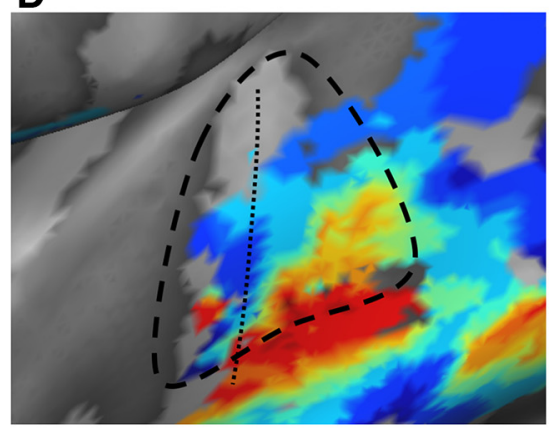

PAC boundary Heschl's gyrus

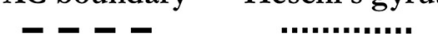

Figure 1. Tonotopic maps in auditory cortex.pRF frequency estimates (for successfully fitted voxels, see Materials and Methods) are shown for the left hemisphere for 2 sighted subjects $(\boldsymbol{A}, \boldsymbol{B})$, and example individuals who were early blind $(\boldsymbol{C})$ or anophthalmic (D). Maps are shown for both the static $(\boldsymbol{A}, \boldsymbol{C})$ and the motion $(\boldsymbol{B}, \boldsymbol{D})$ stimulus. Black dashed line indicates the estimated boundary of PAC for each subject. Black dotted line indicates the location of Heschl's gyrus. To maximize visual similarity, given that the motion stimulus had a smaller frequency range $(100-3162 \mathrm{~Hz})$ than the static stimulus $(88-8000 \mathrm{~Hz})$, the color map is restricted to the frequency range of the motion stimulus. See also Figure 1-1 to Figure 1-29.

revealed a main effect of dataset $\left(F_{(2,46)}=3.92, p=0.0268\right)$, an effect of blindness $\left(F_{(1,46)}=12.8, p=0.0008\right)$, no effect of hemisphere $\left(F_{(1,46)}=0.11, p=0.7468\right)$, and no significant interactions. A post hoc Tukey-Kramer test showed that the UW static dataset resulted in a significantly smaller pRF response amplitudes than the Oxford motion dataset. This could be due to a difference in scanner quality, voxel acquisition size, and/or stimuli.

Within secondary auditory areas, we found no main effect of dataset $\left(F_{(2,46)}=1.62, p=0.2098\right)$, an effect of blindness $\left(F_{(1,46)}=\right.$ $12.33, p=0.001)$, no effect of hemisphere $\left(F_{(1,46)}=0, p=0.9544\right)$, and no significant interactions.

\section{Frequency distributions}

The distribution of frequency preferences within PAC and secondary AC across early blind (red) and sighted subjects (gray) is shown in Figure 3. Individual subjects are shown in Fig. 3-1 and Fig. 3-2. For each dataset, a bootstrapped $\chi^{2}$ test of independence was used to examine the relation between blindness and the number of voxels in each frequency bin ( 6 bins for the motion datasets, 13 bins for the static dataset), by assigning subjects randomly across groups. Using this analysis, within PAC, we saw no effect of blindness on the distribution of frequency preferences for any of the three datasets: Oxford motion, $\chi^{2}(6, N=14)=77.3865, p=0.2710$; UW motion, $\chi^{2}$ $(6, N=8)=57.6207, p=0.3330$; and UW static, $\chi^{2}(13, N=8)=$ $117.6774, p=0.3070$. Within secondary AC, we similarly found no effect of blindness on the distribution of frequency preferences: Ox-

ford motion, $\chi^{2}(6, N=14)=87.5279, p=$ 0.2110; UW motion, $\chi^{2}(6, N=8)=$ $75.7219, p=0.3590$; and UW static, $\chi^{2}$ (13, $N=8)=93.8221, p=0.1870$.

Previous work comparing scanner sequences that differed in their acoustic properties suggests that, although acoustic scanner noise does not result in noticeable systematic misestimation of frequency values near the peak of the scanner noise, it may reduce the number voxels that are successfully fit by the pRF model, thereby biasing the frequency distributions (Thomas et al., 2015). We therefore performed an additional post hoc $\chi^{2}$ analysis of independence examining whether the number of voxels falling inside or outside the $350-2000 \mathrm{~Hz}$ frequency range associated with masking by acoustic noise in the scanner was affected by blindness. Within PAC, we saw an effect of blindness only for the UW static dataset: Oxford motion, $\chi^{2}(1, N=1284)=0.5618, p=0.4535$; UW motion, $\chi^{2}(1, N=1503)=2.0701$, $p=0.1502$; and UW static, $\chi^{2}(1, N=$ $1355)=73.1261, p=0.0000$. Within secondary AC, we found an effect of blindness for all three datasets: Oxford motion, $\chi^{2}(1, N=2062)=11.3067, p=0.0008$; UW motion, $\chi^{2}(1, N=2257)=12.6840$, $p=0.0004$; and UW static, $\chi^{2}(1, N=$ $1898)=10.8173, p=0.0010$. This result might reflect a differential sensitivity to masking effects from scanner noise across blind and sighted populations. However, given that this effect was more consistently observed in secondary AC, it might also reflect a distribution of frequency preferences in blind subjects that is less heavily clustered toward frequencies in the $250-3000 \mathrm{~Hz}$ range (see Discussion).

\section{Tuning width}

Tuning width for each voxel was characterized with $Q$ as given by the following formula:

$$
Q=\frac{f_{0}}{F W H M}
$$

where the FWHM is the estimated Gaussian profile from the pRF model in frequency space. Figure 4 shows $Q$ on the cortical surface in the same example subjects as shown in Figure 1. See also Fig. 1-1 to Fig. 1-29.

To examine differences in tuning width across blind and sighted subjects, we peformed an ANOVA with dataset, blindness, and hemisphere as fixed effects and Q value as the dependent measure. This was done using all successfully fitted voxels for both PAC and auditory cortex.

Within PAC, we found a main effect of dataset $\left(F_{(2,46)}=27.28\right.$, $p<0.0001)$, an effect of blindness $\left(F_{(1,46)}=8.1, p=0.0066\right)$, no effect of hemisphere $\left(F_{(1,46)}=0.56, p=0.4569\right)$, and no significant interactions. A post hoc analysis showed that blindness resulted in significantly larger Q values (narrower tuning) (Fig. 5).

Within secondary auditory areas, we found a main effect of dataset $\left(F_{(2,44)}=29.19, p<0.0001\right)$, no effect of blindness $\left(F_{(1,44)}\right.$ 
A PAC

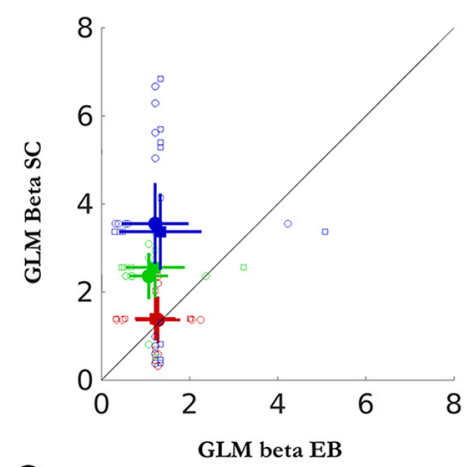

C Secondary AC

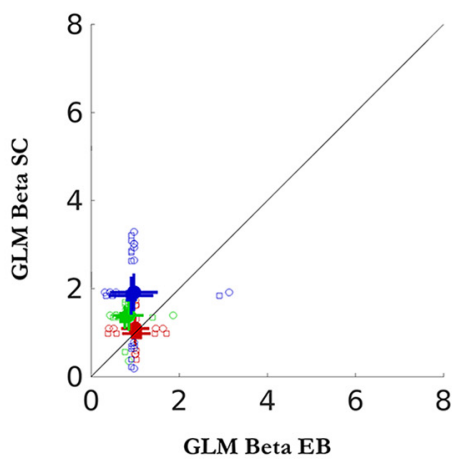

B PAC

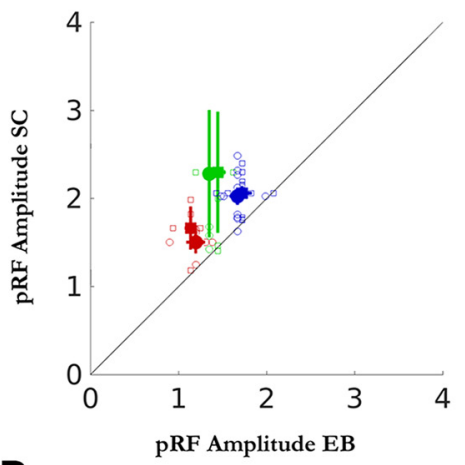

Secondary AC

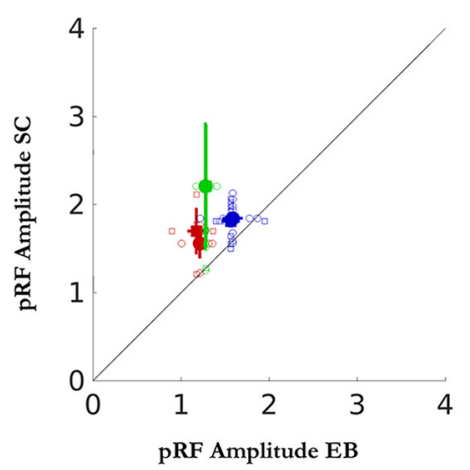

OXFORD MOTION

UW MOTION

UW STATIC

- LEFT HEMISPHERE

- RIGHT HEMISPHERE

Figure 2. Response amplitudes in auditory areas. Blue represents Oxford motion results. Green represents UW motion results. Red represents UW static results. Square and circular symbols represent left and right hemispheres, respectively. $A$, PAC GLM results. $x$ and $y$ axes represent mean blind and sighted subject $\beta$ weights, respectively. $B$, PAC pRF results. $x$ and $y$ axes represent mean blind and sighted subject pRF amplitudes, respectively. $C$, $\boldsymbol{D}$, Secondary auditory cortex GLM and pRF results. Error bars indicate single SEM. See also Figure 2-1 to Figure 2-4.
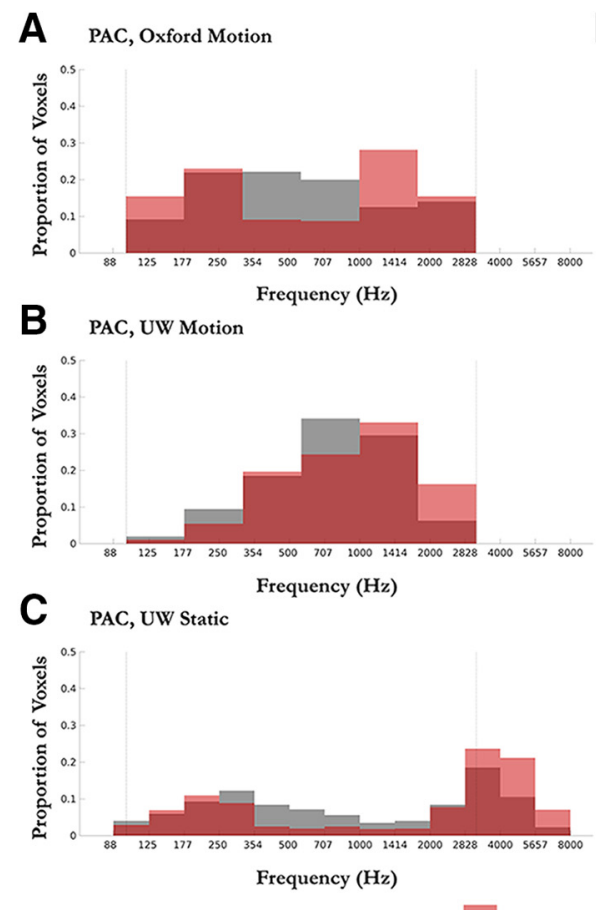

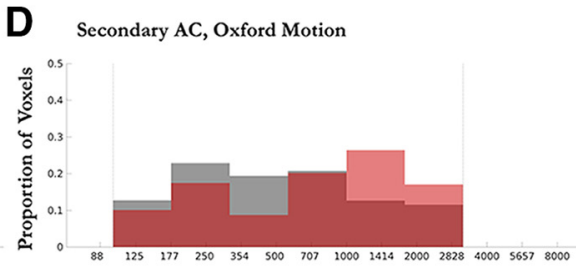

E Secondary AC, UW Motion

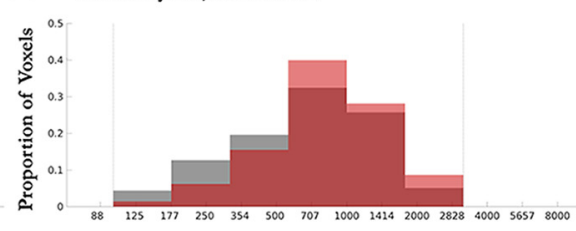

F Secondary AC, UW Static

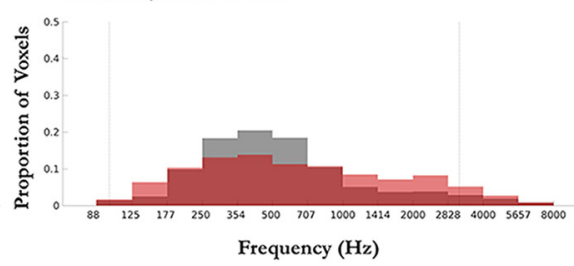

SIGHTED

Figure 3. The proportion of voxels successfully fit using the pRF model as a function of frequency, based on half-octave bins ( 6 bins for the motion datasets, 13 bins for the static dataset). Red represents blind subjects. Gray represents sighted subjects. $\mathbf{A}-\mathbf{C}$, Probability distributions within PAC. D-F, Probability distributions within secondary auditory areas. $A, \mathbf{D}$, Dotted lines indicate the frequency range that was shared across motion and static datasets. See also Figure 3-1 to Figure 3-2. 
A

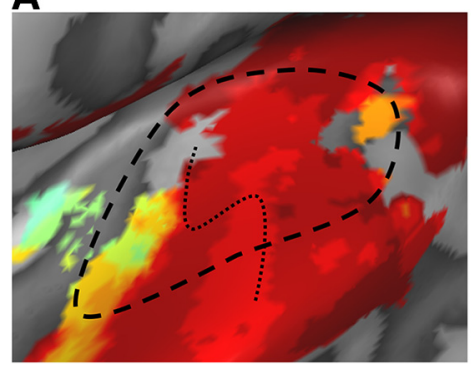

C
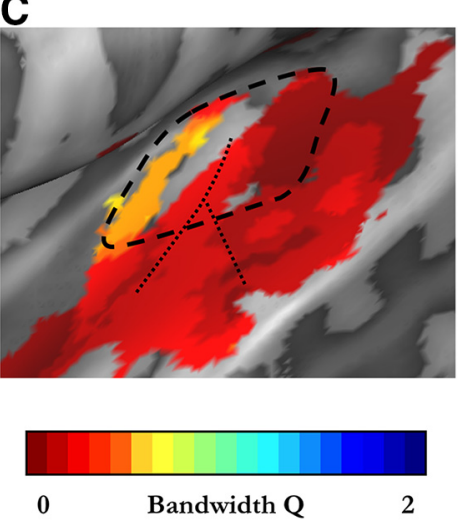

B

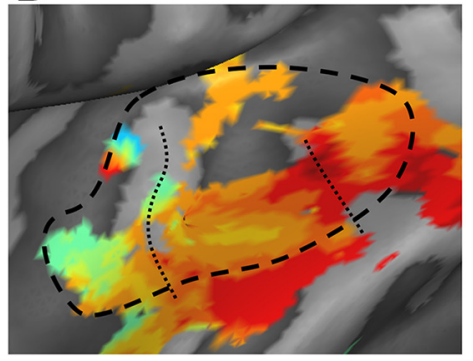

D

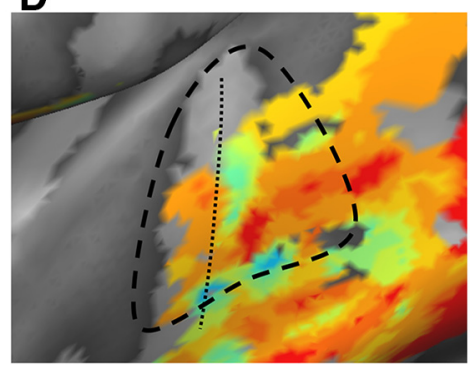

PAC boundary Heschl's gyrus

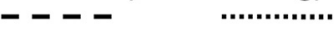

Figure 4. Tuning width maps in auditory cortex. Q estimates (for successfully fitted voxels, see Materials and Methods) are shown for the left hemispherefor 2 sighted subjects $(\boldsymbol{A}, \boldsymbol{B})$, and exampleindividualswho areearlyblind ( $\boldsymbol{C}$ andanophthalmic $(\boldsymbol{D})$. Mapsareshownforboththestatic $(\boldsymbol{A}$, $\boldsymbol{C}$ and the motion $(\boldsymbol{B}, \boldsymbol{D})$ stimulus. Black dashed line indicates the estimated anterior/posterior boundary of PAC for each subject. Black markers represent the location of Heschl's gyrus. See also Figure 1-1 to Figure 1-29.

$=3.55 p=0.066)$, no effect of hemisphere $\left(F_{(1,44)}=1.12, p=\right.$ 0.2954 ), and no significant interactions. The larger $Q$ size in anophthalmic subjects for the Oxford motion stimulus was marginally significant in either PAC and was nonsignificant in secondary auditory cortices, but may reflect a population difference that our sample size was too small to reveal.

For both PAC and secondary auditory cortical areas, post hoc Tukey-Kramer tests showed that the Oxford motion dataset resulted in significantly larger $Q$ values than either of the other two datasets, as can be seen in Figure 5. This is likely due to the smaller acquisition voxel size in this dataset because a smaller voxel presumably reflects a more homogeneous neural population of tuning preferences (Dumoulin and Wandell, 2008).

Visual inspection and statistical analyses did not reveal any consistent relationship between tuning width ( $\mathrm{Q}$ values) and frequency that was reliable across datasets, or reliably different across blind and sighted subjects (see Fig. 5-1 and Fig. 5-2).

Estimated population tuning widths are presumably influenced both by the breadth of underlying individual neural tuning curves and by the dispersion of frequency preferences within each voxel. For each voxel, we estimated "frequency dispersion" as the median difference in center frequency between that voxel and all adjacent voxels, normalized by the Euclidean distance in millimeters between the voxels on the cortical surface. Group differences were assessed using an ANOVA with dataset, hemisphere, and blindness as fixed effects and dispersion within PAC as the dependent measure. Within PAC, we found a main effect of dataset $\left(F_{(2,45)}=71.73, p<0.0001\right)$, an effect of blindness $\left(F_{(1,45)}=\right.$ $5.79, p=0.0203)$, no effect of hemisphere $\left(F_{(1,45)}=0.21, p=\right.$ $0.6527)$, and a significant interaction between dataset and blindness $\left(F_{(2,45)}=3.43, \mathrm{p} 0.0411\right)$. No other interactions were significant. Within secondary auditory areas, we found a main effect of dataset $\left(F_{(2,46)}=101.38, p<0.0001\right)$, no effect of blindness
$\left(F_{(1,46)}=0.39 p=0.5353\right)$, no effect of hemisphere $\left(F_{(1,46)}=0.01, p=0.9046\right)$, and no significant interactions.

Finally, we examined whether differences in $\mathrm{Q}$ within PAC could be explained by differences in frequency dispersion using multivariate linear regression with dataset, hemisphere, frequency dispersion, and blindness as fixed effects and $\mathrm{Q}$ as the dependent measure. As might be expected, frequency dispersion was a strong predictor of $\mathrm{Q}(b=-1.5799, p<0.0001)$, with high dispersion values predicting low $\mathrm{Q}$ values. However, we still found significantly higher $\mathrm{Q}$ values in blind individuals $(b=0.1446, p<0.0001)$, even after including frequency dispersion as an independent factor.

\section{Discussion}

Here we examined whether blindness early in life alters the representation of frequency information within auditory cortex. Using an adaptation of the population receptive field model, we were able to disentangle voxel level tuning widths from response amplitudes. We find evidence that early blindness results in narrower bandwidths, reduced pRF amplitudes, and may alter the distribution of frequency preferences within auditory cortex. We did not see any effect of blindness on the size or the hemodynamic responsivity of PAC and secondary AC.

\section{Auditory cortex size}

Here, we failed to find evidence for an expanded tonotopic representation of PAC or secondary auditory areas as suggested by previous MEG data (Elbert et al., 2002). One possibility is that the apparent increase in source separation of high and low frequencies in early blind subjects that was noted previously by Elbert et al. (2002) was driven by differences in tuning bandwidth. Broader bandwidths in sighted subjects would be expected to result in a correspondingly larger cortical area of activation for any given auditory frequency. This might, in turn, have reduced the apparent source separation of high and low frequencies (Golubic et al., 2011). Moreover, the known variability in cortical folding within Heschl's gyrus (Da Costa et al., 2011) would be expected to complicate estimates of PAC size, especially given that dipole estimates in the study by Elbert et al. (2002) were based on a best fitting local sphere rather than individual anatomies. However, it remains possible that a future study with a larger sample might reveal subtle differences in the structure of PAC and/or secondary AC. Indeed, Atilgan et al. (2017) recently reported reduced surface area in subregions of secondary auditory cortex for congenitally blind subjects, along with evidence for greater bilateral similarity between cortical thickness and surface area in both early and late blind subjects throughout the entire superior temporal plane.

\section{Response amplitudes}

A number of previous studies have reported an attenuated response to pure tone stimuli versus silence in the temporal lobe of 
blind individuals (Gougoux et al., 2009; Stevens and Weaver, 2009; Watkins et al., 2013) when comparing responses to pure tones versus silence. Reduced responses have been interpreted as reduced participation in auditory processing, perhaps due to increased "efficiency" of processing within the intact modality or due to function being "usurped" by a reorganized occipital cortex (Jiang et al., 2014; Dormal et al., 2015). One concern is that these previous results might have reflected narrower tuning rather than reduced responsiveness because narrower tuning would be expected to result in a smaller of region of cortex responding to any given narrowband stimulus, which would reduce the measured activation in a sound versus silence GLM. If blind individuals have neurons that are more narrowly tuned for more complex spectrotemporal modulations (more specialized "feature detectors"), this would reduce the population response to any given pure tone or bandpass stimulus. It is known that secondary auditory areas contain neurons that have multidimensional tuning that reflect complex spatiotemporal properties of the stimulus (Schönwiesner and Zatorre, 2009; Moerel et al., 2013, 2018; Santoro et al., 2014, 2017; Allen et al., 2018; De Angelis et al., 2018), and previous work suggests an increase in the proportion of spatially tuned cells within anterior auditory association areas in visually deprived cats (Korte and Rauschecker, 1993). An advantage of our pRF approach is that it allows an independent representation tuning width and response amplitude. Here, we replicated previous findings showing reduced $\beta$ weights, and similarly found reduced $\mathrm{pRF}$ amplitudes as a result of early blindness. We then separately examined frequency tuning bandwidth in each group, as described below.

\section{Frequency distributions}

Previous work in monkeys has shown that frequency representations within PAC can be altered by experience (Recanzone et al., 1992, 1993), with a shift toward trained frequencies. Given that blind subjects rely on auditory frequency for a wider range of tasks than sighted individuals, we thought it possible that we might see a difference in frequency representations across the two groups. In our initial analysis, we did not see evidence for an alteration in the distribution of frequency preferences as a result of blindness within either PAC or secondary auditory areas. However, a post hoc analysis revealed fewer voxels falling in the 350-2000 $\mathrm{Hz}$ range within PAC for the UW static dataset, and for all three datasets within secondary AC. This result might reflect a differential sensitivity to masking effects of acoustic scanner noise across blind and sighted populations. However, given that this effect was more consistently observed in secondary AC, it might also reflect a distribution of frequency preferences in blind subjects that is less heavily clustered toward frequencies that fall in the $250-3000 \mathrm{~Hz}$ range, perhaps driven by the use of auditory cues with broad spectral content, such as acoustic echoes produced by mouth sounds or a cane (Norman and Thaler, 2017).
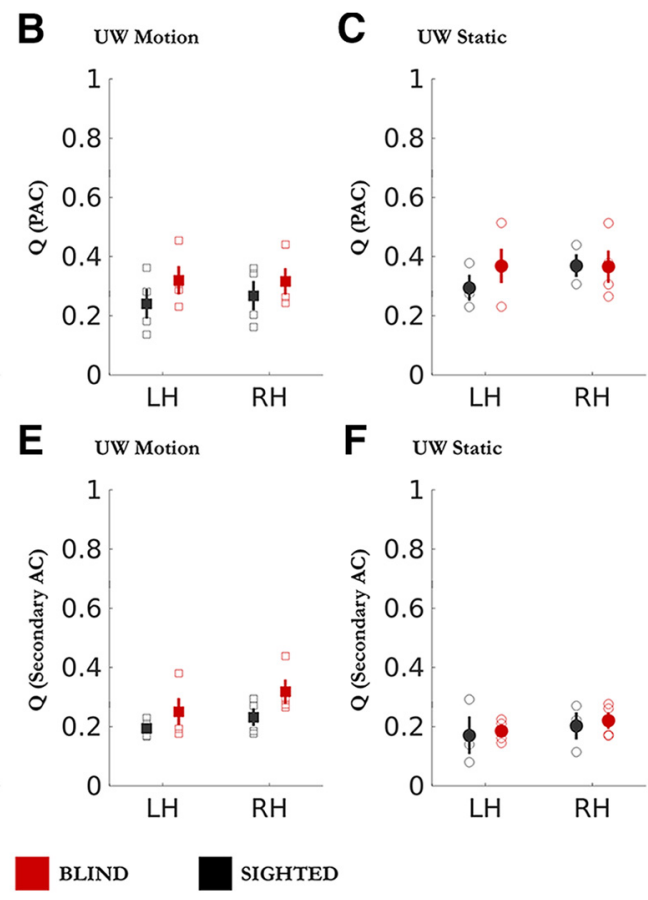

\section{BLIND}

ary auditory cortex (D-F) for left (LH) and right (RH) hemispheres. Mean pRF size was calculated for each subject. Symbols represent group means with single SEMs calculated (RH) hemispheres. Mean pRF size was calculated for
across subjects. See also Figure 5-1 to Figure 5-2.

\section{Tuning width}

Blind individuals had significantly narrower voxelwise tuning for auditory frequency within both left and right PAC. We do not believe that the narrowing in pRF tuning width within PAC that we observed in blind individuals was due to differences in the gradient of preferred frequency across the cortical surface. As described above, both PAC/auditory cortex size and the dispersion of frequencies within PAC were similar across both subject groups, and visual inspection revealed no systematic gradient differences. Nor, given the similarity in the measured hemodynamic responses between our subject groups, do we believe that these differences are due to group differences in hemodynamic coupling. It seems more likely that our PAC results either reflect (1) a narrowing in the tuning bandwidth of individual neurons or (2) a more refined local organization, such as a reduction in the amount of scatter in frequency preference across a scale of $<3 \mathrm{~mm}$. Consistent with the notion that these differences might reflect differences in neural tuning within individual neurons, Petrus et al. (2014) have shown that adult-onset visual deprivation over 6-8 d sharpens the frequency tuning of individual neurons within A1 in the mouse. Moreover, the same brief period of visual deprivation leads to more refined interlaminar connections (Meng et al., 2015, 2017), highlighting the capability for rapid remodeling of auditory frequency representations, even after the closure of the canonical critical period.

We did not see differences is tuning bandwidth within secondary AC as a result of early blindness. However, we used relatively simple stimuli and a simple Gaussian pRF model, and these areas are known to have complex spectrotemporal tuning functions (Schönwiesner and Zatorre, 2009; Barton et al., 2012; Moerel et al., 2013, 2014, 2018; Santoro et al., 2014, 2017). Future work, using more naturalistic stimuli and more complex analysis models, will be important for more fully 
characterizing the effects of blindness on auditory tuning in these secondary areas.

In conclusion, here we provide some of the first evidence for systematic changes in neural tuning within human auditory cortex as a result of blindness. It remains to be seen whether the changes described here reflect a developmental adaptation to early blindness, the ongoing effects of visual deprivation, and/or differential auditory demands that result from being blind. Future work could examine these questions by addressing whether adult-onset blindness, short-term visual deprivation, and/or auditory training can alter frequency tuning within auditory cortex, and whether, in adult sight-recovery subjects, the effects of longterm visual deprivation on auditory cortex are reversed with the reinstatement of vision.

\section{References}

Allen EJ, Moerel M, Lage-Castellanos A, De Martino F, Formisano E, Oxenham AJ (2018) Encoding of natural timbre dimensions in human auditory cortex. Neuroimage 166:60-70.

Atilgan H, Collignon O, Hasson U (2017) Structural neuroplasticity of the superior temporal plane in early and late blindness. Brain Lang 170:71-81.

Barton B, Venezia JH, Saberi K, Hickok G, Brewer AA (2012) Orthogonal acoustic dimensions define auditory field maps in human cortex. Proc Natl Acad Sci U S A 109:20738-20743.

Boynton GM, Engel SA, Glover GH, Heeger DJ (1996) Linear systems analysis of functional magnetic resonance imaging in human V1. J Neurosci 16:4207-4221.

Coullon GS, Emir UE, Fine I, Watkins KE, Bridge H (2015) Neurochemical changes in the pericalcarine cortex in congenital blindness attributable to bilateral anophthalmia. J Neurophysiol 114:1725-1733.

Da Costa S, van der Zwaag W, Marques JP, Frackowiak RS, Clarke S, Saenz M (2011) Human primary auditory cortex follows the shape of Heschl's gyrus. J Neurosci 31:14067-14075.

Da Costa S, Saenz M, Clarke S, van der Zwaag W (2015) Tonotopic gradients in human primary auditory cortex: concurring evidence from highresolution $7 \mathrm{~T}$ and $3 \mathrm{~T}$ fMRI. Brain Topogr 28:66-69.

De Angelis V, De Martino F, Moerel M, Santoro R, Hausfeld L, Formisano E (2018) Cortical processing of pitch: model-based encoding and decoding of auditory fMRI responses to real-life sounds. Neuroimage 180:291-300.

De Volder AG, Bol A, Blin J, Robert A, Arno P, Grandin C, Michel C, Veraart C (1997) Brain energy metabolism in early blind subjects: neural activity in the visual cortex. Brain Res 750:235-244.

Dick F, Tierney AT, Lutti A, Josephs O, Sereno MI, Weiskopf N (2012) In vivo functional and myeloarchitectonic mapping of human primary auditory areas. J Neurosci 32:16095-16105.

Dormal G, Lepore F, Harissi-Dagher M, Albouy G, Bertone A, Rossion B, Collignon O (2015) Tracking the evolution of crossmodal plasticity and visual functions before and after sight restoration. J Neurophysiol 113: $1727-1742$

Dumoulin SO, Wandell BA (2008) Population receptive field estimates in human visual cortex. Neuroimage 39:647-660.

Elbert T, Sterr A, Rockstroh B, Pantev C, Muller MM, Taub E (2002) Expansion of the tonotopic area in the auditory cortex of the blind. J Neurosci 22:9941-9944.

Formisano E, Kim DS, Di Salle F, van de Moortele PF, Ugurbil K, Goebel R (2003) Mirror-symmetric tonotopic maps in human primary auditory cortex. Neuron 40:859-869.

Golubic SJ, Susac A, Grilj V, Ranken D, Huonker R, Haueisen J, Supek S (2011) Size matters: MEG empirical and simulation study on source localization of the earliest visual activity in the occipital cortex. Med Biol Eng Comput 49:545-554.

Gougoux F, Lepore F, Lassonde M, Voss P, Zatorre RJ, Belin P (2004) Neuropsychology: pitch discrimination in the early blind. Nature 430:309.

Gougoux F, Belin P, Voss P, Lepore F, Lassonde M, Zatorre RJ (2009) Voice perception in blind persons: a functional magnetic resonance imaging study. Neuropsychologia 47:2967-2974.

Griswold MA, Jakob PM, Heidemann RM, Nittka M, Jellus V, Wang J, Kiefer B, Haase A (2002) Generalized autocalibrating partially parallel acquisitions (GRAPPA). Magn Reson Med 47:1202-1210.
Hackett TA (2008) Anatomical organization of the auditory cortex. J Am Acad Audiol 19:774-779.

Hackett TA, Stepniewska I, Kaas JH (1998) Subdivisions of auditory cortex and ipsilateral cortical connections of the parabelt auditory cortex in macaque monkeys. J Comp Neurol 394:475-495.

Huber E, Jiang F, Fine I (2019) Responses in area $\mathrm{hMT}^{+}$reflect tuning for both auditory frequency and motion after blindness early in life. Proc Natl Acad Sci U S A. Advance online publication. Retrieved April 29, 2019. doi: 10.1073/ pnas. 1815376116.

Humphries C, Liebenthal E, Binder JR (2010) Tonotopic organization of human auditory cortex. Neuroimage 50:1202-1211.

Jiang F, Stecker GC, Fine I (2014) Auditory motion processing after early blindness. J Vis 14:4.

Korte M, Rauschecker JP (1993) Auditory spatial tuning of cortical neurons is sharpened in cats with early blindness. J Neurophysiol 70:1717-1721.

Kujala T, Alho K, Paavilainen P, Summala H, Näätänen R (1992) Neural plasticity in processing of sound location by the early blind: an eventrelated potential study. Electroencephalogr Clin Neurophysiol 84:469472.

Kujala T, Huotilainen M, Sinkkonen J, Ahonen AI, Alho K, Hämäläinen MS, Ilmoniemi RJ, Kajola M, Knuutila JE, Lavikainen J (1995) Visual cortex activation in blind humans during sound discrimination. Neurosci Lett 183:143-146.

Langers DR, van Dijk P (2012) Mapping the tonotopic organization in human auditory cortex with minimally salient acoustic stimulation. Cereb Cortex 22:2024-2038.

Meng X, Kao JP, Lee HK, Kanold PO (2015) Visual deprivation causes refinement of intracortical circuits in the auditory cortex. Cell Rep 12:955964.

Meng X, Kao JP, Lee HK, Kanold PO (2017) Intracortical circuits in thalamorecipient layers of auditory cortex refine after visual deprivation. eNeuro 4:ENEURO.0092-17.2017.

Moeller S, Yacoub E, Olman CA, Auerbach E, Strupp J, Harel N, Ugurbil K (2010) Multiband multislice GE-EPI at 7 tesla, with 16-fold acceleration using partial parallel imaging with application to high spatial and temporal whole-brain fMRI. Magn Reson Med 63:1144-1153.

Moerel M, De Martino F, Formisano E (2012) Processing of natural sounds in human auditory cortex: tonotopy, spectral tuning, and relation to voice sensitivity. J Neurosci 32:14205-14216

Moerel M, De Martino F, Santoro R, Ugurbil K, Goebel R, Yacoub E, Formisano E (2013) Processing of natural sounds: characterization of multipeak spectral tuning in human auditory cortex. J Neurosci 33:11888-11898.

Moerel M, De Martino F, Formisano E (2014) An anatomical and functional topography of human auditory cortical areas. Front Neurosci 8:225.

Moerel M, De Martino F, Kemper VG, Schmitter S, Vu AT, Ugurbil K, Formisano E, Yacoub E (2018) Sensitivity and specificity considerations for fMRI encoding, decoding, and mapping of auditory cortex at ultra-high field. Neuroimage 164:18-31.

Norman L, Thaler L (2017) Human echolocation-spatial resolution and signal properties. London: Institution of Engineering and Technology.

Petrus E, Isaiah A, Jones AP, Li D, Wang H, Lee HK, Kanold PO (2014) Crossmodal induction of thalamocortical potentiation leads to enhanced information processing in the auditory cortex. Neuron 81:664-673.

Rademacher J, Morosan P, Schormann T, Schleicher A, Werner C, Freund HJ, Zilles K (2001) Probabilistic mapping and volume measurement of human primary auditory cortex. Neuroimage 13:669-683.

Recanzone GH, Jenkins WM, Hradek GT, Merzenich MM (1992) Progressive improvement in discriminative abilities in adult owl monkeys performing a tactile frequency discrimination task. J Neurophysiol 67: 1015-1030.

Recanzone GH, Schreiner CE, Merzenich MM (1993) Plasticity in the frequency representation of primary auditory cortex following discrimination training in adult owl monkeys. J Neurosci 13:87-103.

Santoro R, Moerel M, De Martino F, Goebel R, Ugurbil K, Yacoub E, Formisano E (2014) Encoding of natural sounds at multiple spectral and temporal resolutions in the human auditory cortex. PLoS Comput Biol 10: e1003412.

Santoro R, Moerel M, De Martino F, Valente G, Ugurbil K, Yacoub E, Formi- 
sano E (2017) Reconstructing the spectrotemporal modulations of reallife sounds from fMRI response patterns. Proc Natl Acad Sci U S A 114:4799-4804

Schönwiesner M, Zatorre RJ (2009) Spectro-temporal modulation transfer function of single voxels in the human auditory cortex measured with high-resolution fMRI. Proc Natl Acad Sci U S A 106:14611-14616.

Stevens AA, Weaver KE (2009) Functional characteristics of auditory cortex in the blind. Behav Brain Res 196:134-138.

Striem-Amit E, Hertz U, Amedi A (2011) Extensive cochleotopic mapping of human auditory cortical fields obtained with phase-encoding FMRI. PLoS One 6:e17832.

Thomas JM, Huber E, Stecker GC, Boynton GM, Saenz M, Fine I (2015) Population receptive field estimates of human auditory cortex. Neuroimage 105:428-439.

Veraart C, De Volder AG, Wanet-Defalque MC, Bol A, Michel C, Goffinet AM (1990) Glucose utilization in human visual cortex is abnormally elevated in blindness of early onset but decreased in blindness of late onset. Brain Res 510:115-121.

Voss P, Zatorre RJ (2012) Occipital cortical thickness predicts performance on pitch and musical tasks in blind individuals. Cereb Cortex 22: 2455-2465.

Wan CY, Wood AG, Reutens DC, Wilson SJ (2010) Early but not late-blindness leads to enhanced auditory perception. Neuropsychologia 48:344-348.

Wanet-Defalque MC, Veraart C, De Volder A, Metz R, Michel C, Dooms G, Goffinet A (1988) High metabolic activity in the visual cortex of early blind human subjects. Brain Res 446:369-373.

Watkins KE, Shakespeare TJ, O'Donoghue MC, Alexander I, Ragge N, Cowey A, Bridge $\mathrm{H}$ (2013) Early auditory processing in area V5/MT ${ }^{+}$of the congenitally blind brain. J Neurosci 33:18242-18246.

Weaver KE, Richards TL, Saenz M, Petropoulos H, Fine I (2013) Neurochemical changes within human early blind occipital cortex. Neuroscience 252:222-233. 